\title{
Dissociations between temporally cued and thematically cued recall
}

\author{
RONALD P. FISHER \\ Florida International University, Miami, Florida \\ and \\ CARLA C. CHANDLER \\ University of Toronto, Toronto, Ontario, Canada
}

\begin{abstract}
Subjects listened to paired comparisons that conformed to an underlying order or theme (cf. Potts, 1975). Immediately afterward or $30 \mathrm{~min}$ later, either temporal or thematic cues were given for recall of these items. Typical serial position effects were found only for temporally cued recall. By comparison, position along the underlying theme affected recall accuracy only when recall was cued thematically. In addition, temporally cued recall showed the expected decrease in accuracy with delayed recall. Thematically cued recall, however, showed a reminiscence effect: Recall was better after the 30-min delay than it was immediately after presentation. These findings were interpreted as support for a distinction between episodic and thematic codings of the items. The reminiscence found in thematically cued recall was explained in terms of strategic use of the hypothesized episodic and thematic codes.
\end{abstract}

Current theorizing in memory suggests that recollection of an event may occur for either of two reasons. First, when an event occurs, a trace of that specific event is established. Later activation of the trace leads to recollection of the event. Second, when many related events occur, they are represented by a general theme which reflects the commonality across events. Recollection of a given event is then inferred or constructed from the theme. We refer to the first form of memory as episodic retrieval and to the second as thematic construction (Fisher \& Cuervo, 1983; Hasher \& Griffin, 1978; Reder, 1982).

Does memory mediated by episodic retrieval differ from that mediated by thematic construction? One way to examine this issue is to compare recall of related events, for which thematic construction may be used, with recall of unrelated events, for which only episodic retrieval is appropriate. Fisher and Cuervo (1983) found that recall of unrelated experiences is more heavily influenced by the physical characteristics of the acquisition events than is recall of theme-related events. In addition, Fisher, Bixby, and Oliver (1981) observed that the temporal relations across events are more pronounced for unrelated events than for theme-related events (see also, Smith, Adams, \& Schorr, 1978).

Additional evidence for a distinction between episodic retrieval and thematic construction derives from studies that have presented only thematically consistent events. Here, it is assumed that different experimental

The first author's mailing address is: Department of Psychology, Florida International University, Miami, FL 33199. The second author's mailing address is: Department of Psychology, University of Toronto, Toronto, Ontario, Canada M5S 1A1. tasks reflect the two forms of memory. Typically, recognition of individual items is used to measure episodic retrieval, whereas categorization and judgments of thematic consistency are used to measure thematic construction. Evidence for two forms of memory comes from the finding that some experimental manipulations have different effects on the two tasks. For example, recognition accuracy is better than categorization accuracy when testing is immediate, but the opposite is true when testing is delayed (e.g., Hock \& Schmelzkopf, 1980; Posner \& Keele, 1968). Similarly, Reder (1982) found that at immediate testing, latency to recognize a sentence is faster than judging its consistency with a theme, but at delayed testing, the relation reverses.

In the present study, we again show that recollections of individual events and of themes are dissociable phenomena. We presented a series of thematically related items (cf. Potts, 1975) and then provided recall cues that referred to an item either by its temporal or by its thematic characteristics. We expected a double dissociation, whereby serial input position would affect only temporally cued recall and thematic position would affect only thematically cued recall.

\section{METHOD}

\section{Procedure and Materials}

The subjects were told that they would hear several facts about animals fighting for dominion over land. Each fact would compare the intelligence of two animals, and an animal's success in battle would be determined by its relative intellect. The subjects were informed that a test would follow.

The experimenter then presented nine statements of the form: The (Animal A) is smarter than the (Animal B). The statements were read in a normal speaking voice, with $10 \mathrm{sec}$ separating the end of one statement and the beginning of the 
next. All nine statements were consistent with an arbitrarily designated order of six animals (elephant, giraffe, deer, goat, tiger, kangaroo). There were three critical statements, which compared the two most intelligent animals, the two least intelligent animals, and the two animals of intermediate intelligence. These three statements were always presented as the first, fifth, and ninth statements. Assignment of the three critical statements to input positions 1,5 , and 9 was counterbalanced across subjects. The remaining six statements were assigned randomly among the remaining input positions.

The subjects were tested either immediately (15 sec after the last statement) or after a 30-min filled or unfilled delay. In the unfilled delay condition, the subjects were given $30 \mathrm{~min}$ of free time, with the constraints that they remain in the experimental room and not write down or discuss anything concerning the experiment. In the filled delay condition, the subjects performed a Stroop task for $30 \mathrm{sec}$, an arithmetic task for $2 \mathrm{~min}$, and then an unrelated learning task for the remaining $27.5 \mathrm{~min}$.

All of the subjects were tested for their recall of the three critical statements, comparing the two most intelligent, the two intermediately intelligent, and the two least intelligent animals. In each delay condition, half of the subjects were given three ordinal retrieval cues that referred to the critical statements, "The first (fifth, last) fact was: The is smarter than the ___ The remaining subjects were given three thematic retrieval cues that referred to the same statements, "Of the two most (intermediate, least) intelligent animals: The smarter than the ." Each statement was tested first, second, and third equally often across subjects. The subjects were instructed to fill in the blanks with the appropriate animal names for all three questions and, if unsure, to guess. The test was subject paced.

\section{Subjects and Design}

The subjects were 432 UCLA undergraduates, who participated in the experiment to fulfill a course requirement. The subjects were assigned randomly to one of six experimental groups, resulting in 72 subjects per group. Each group represented a unique combination of retrieval cue (ordinal, thematic) and retention interval (immediate, unfilled delay, filled delay).

In each group, the subjects were tested for recall of the same three facts. These three facts, which compared the two most, two intermediately, and two least intelligent animals, had been presented equally often as the first, fifth, and ninth statements. Thus, recall could be examined as a function of serial input position, summing across thematic positions. Similarly, recall could be examined as a function of thematic position, summing across serial input positions.

\section{RESULTS}

A response was scored as correct only if both animals were recalled and in the appropriate order. (A second analysis, not shown here, revealed the same patterns using a lenient scoring system, in which one point was awarded for each animal recalled, regardless of order.) For clarity, the results are presented in two parts. First, recall is examined as a function of retention interval and retrieval cue. Second, the effects of retention interval and retrieval cue are examined as a function of serial input position and thematic position. All results are reported at the $p<.05$ level, unless otherwise indicated.

An ANOVA showed a reliable main effect of retrieval cue $[F(1,426)=23.19]$ and an unreliable main effect of retention interval $[F(2,426)=1.88]$. More important, there was a reliable interaction between retrieval cue and retention interval $[F(2,426)=11.19]($ all MSes $=.23)$. Retention interval had opposite effects on ordinally
Table 1

Probability of Correct Recall as a Function of Retrieval Cue and Retention Interval

\begin{tabular}{cccc} 
& \multicolumn{3}{c}{ Retention Interval } \\
\cline { 2 - 4 } Retrieval Cue & Immediate & $\begin{array}{c}\text { Unfilled } \\
\text { Delay }\end{array}$ & $\begin{array}{c}\text { Filled } \\
\text { Delay }\end{array}$ \\
\hline Ordinal & .51 & .35 & .32 \\
Thematic & .20 & .32 & .27 \\
\hline
\end{tabular}

cued and thematically cued recall (see Table 1). For the ordinal cue, recall was better immediately afterward than following an unfilled (Newman-Keuls: $q=8.62$ ) or filled $(q=10.10)$ delay. For the thematic cue, however, recall was reliably worse immediately afterward than following an unfilled $(q=6.16)$ or filled $(q=3.45)$ delay.

The second set of analyses showed how the effects of retrieval cue and retention interval varied with serial input position and with thematic position. The upper panels of Figure 1 show that ordinally cued recall changed as a function of serial input position, whereas thematically cued recall was relatively unaffected. For the ordinal retrieval cue, immediate recall showed a typical U-shaped function, whereas delayed recall showed only a primacy effect. In contrast, thematically cued recall was flat across serial positions. A three-way ANOVA indicated a reliable main effect of serial position $[F(2,852)=92.13]$, two reliable two-way interactions [retrieval cue $\mathrm{x}$ serial position, $\mathrm{F}(2,852)=61.97$; retention interval $x$ serial position $F(2,852)=8.99$ ], and a reliable three-way interaction [retrieval cue $\mathrm{x}$ retention interval $x$ serial position, $F(4,852)=2.87$ ] (all MSes $=.15$ ).

The lower panels of Figure 1, which describe the effects of retrieval cue and retention interval as a function of thematic position, depict a similar dissociation. Thematically cued recall varied markedly with thematic

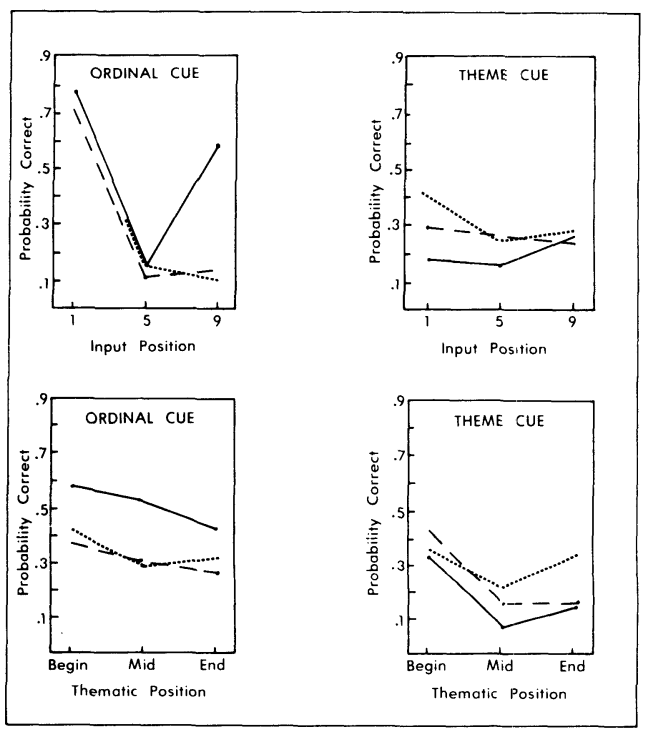

Figure 1. Recall accuracy for ordinal and theme cues as a function of serial input position and thematic position (immediate: $\bullet$; unfilled delay: $\cdots \cdots$; filled delay: $\bullet-\longrightarrow$ ). 
position, whereas the effect was much weaker for ordinally cued recall. Generally, thematically cued recall followed a U-shaped curve: better recall of the most and least intelligent than of the intermediately intelligent animals. In contrast, ordinally cued recall showed little change across thematic position. An ANOVA revealed a reliable main effect of thematic position $[F(2,852)=$ $15.85]$ and a marginally reliable interaction between retrieval cue and thematic position $[\mathrm{F}(2,852)=2.49$, $\mathrm{p}<.09$ ]. No other effects were reliable (Fs $<1.05$, MSes $=.20$ ).

\section{DISCUSSION}

The pattern of data in the present experiment extends earlier work showing that item and theme recollection are qualitatively different. Whereas ordinal cuing yielded better recall in some test conditions, theme cuing was better in other conditions. For this reason, we suggest that it is not meaningful to compare the efficacy of episodic trace retrieval and thematic construction on a global level (cf. Camp, Lachman, \& Lachman, 1980; Reder, 1982). Instead, it is more instructive to examine the dissociation between these retrieval modes and experimental manipulations. In the present experiment, two variables differentially affected ordinally cued and thematically cued recall-serial input position and thematic position. Serial input position affected only ordinally cued recall, whereas thematic position affected only thematically cued recall.

We suggest tentatively that the reason for the double dissociation is that recall of specific items and recall of themes are based on different codes (Fisher \& Chandler, 1984). Codes used in episodic retrieval represent the features that define an item as a unique event. The specific code, then, reflects the physical, semantic, and temporal properties of each item. Thus, items that are temporally distinctive (recent items in immediate recall) are well remembered when the retrieval is mediated by the episodic system. When the temporal distinctiveness of these items is diminished, by imposing a long retention interval (Bellezza, 1982; Bjork \& Whitten, 1974), their episodic retrieval suffers. By comparison, the thematic code reflects only what is semantically common across many items. The physical and temporal characteristics that uniquely describe each item are not represented. As such, thematically cued recall does not reflect the temporal distinctiveness of a given item. In the present experiment, recall to the thematic cue never showed a recency effect, even at immediate recall.

An alternative to the suggested two-code system is that there is only one underlying code, but that there are two different retrieval processes applied in the two experimental tasks (Reder, 1982). Although the present experiment did not allow us to distinguish between these two alternatives, some recent findings by Fisher and Chandler (1984) support a two-code approach. In their study, a subject was tested for recall of the same item on two successive occasions. Initial recall facilitated final recall only when both tests provided similar cues (both episodic or both theme cues). There was no facilitation for theme-cued recall of an item if an episodic cue was given for initial recall, and vice versa. Furthermore, successive recalls of the same item were stochastically independent when different retrieval cues were given on the two tests. It thus appears that the underlying codes that mediate episodic retrieval and theme retrieval are independent.

It should be noted that one temporal variable, retention interval, did influence theme-cued recall. There was a reliable increase in accuracy from immediate to delayed recall. Such reminiscence, especially when different subjects are examined at short and long retention intervals, is extremely rare (Burns, 1981). The effect appears not to be the result of more conscious rehearsal during the retention interval, since the effect occurred in both filled and unfilled delay conditions. We would like to suggest that there is an inhibiting factor present at immediate recall that becomes ineffective at delayed recall. Specifically, we suggest that at the immediate test, the last episodic code is highly accessible, so that the subject is enticed to retrieve this code. Although retrievable, the episodic code is not particularly useful for recall when the thematic cue is given. Retrieving episodes, however, inhibits using the more appropriate thematic code. At longer retention intervals, the episodic code becomes less accessible, and therefore less likely to be used. Consequently, the thematic code, the more appropriate code, is more likely to be used at longer retention intervals.

Although the foregoing explanation is obviously post hoc, it is surprisingly similar to one proposed independently by Reder (1982) to explain a comparable reminiscence effect with plausibility judgments. Reder found that, after listening to a short story, subjects judged the plausibility of a test sentence faster if it was presented after a 2-day delay than if it was presented immediately. She suggested that at the immediate test, lexical traces are highly accessible, so that subjects are enticed to retrieve these traces. At longer retention intervals, when lexical traces are less accessible, the subjects use a more appropriate retrieval strategy for plausibility judgments. For the moment, we present this inhibition explanation of the reminiscence effect to account only for the data found in the present experiment. The phenomenon of reminiscence is rare. However, its occurrence may have considerable heuristic value.

\section{REFERENCES}

Bellezza, F. S. (1982). Updating memory using mnemonic devices. Cognitive Psychology, 14, 301-327.

Bjork, R. A., \& WhitTen, W. B. (1974). Recency-sensitive retrieval processes in long-term free recall. Cognitive Psychology, 6, 173-189.

BURNS, M. J. (1981). The mental retracing of prior real-world actions. Unpublished doctoral dissertation, University of California, Los Angeles.

Camp, C. J., Lachman, J. L., \& Lachman, R. (1980). Evidence for direct-access and inferential retrieval in question-answering. Journal of Verbal Learning and Verbal Behavior, 19, 583-596.

Fisher, R. P., Bixby, D. T., \& Oliver, R. (1981, November). Constructive memory vs. trace retrieval: Some of each. Paper presented at the meeting of the Psychonomic Society, Philadelphia.

Fisher, R. P., \& Chandler, C. C. (1984). The independence of theme-cued and speaker-cued recall. Unpublished manuscript, Florida International University.

Fisher, R. P., \& Cuervo, A. (1983). Memory for physical features of discourse as a function of their relevance. Journal of Experimental Psychology: Learning, Memory, and Cognition, 9, 130-138.

Hasher, L., \& Griffin, M. (1978). Reconstructive and reproductive processes in memory. Journal of Experimental Psychology: Human Learning and Memory, 4, 318-330.

Hock, H. S., \& Schmelzkopf, K. F. (1980). The abstraction of schematic representations from photographs of real-world scenes. Memory \& Cognition, 8, 543-554.

Posner, M. I., \& Keele, S. W. (1968). On the genesis of abstract ideas. Journal of Experimental Psychology, 77, 353-363.

PotTs, G. R. (1975). Bringing order to cognitive structures. In F. Restle, R. Shiffrin, N. Castellan, H. Lindman, \& D. Pisoni (Eds.), Cognitive theory (Vol. 1). Hillsdale: Erlbaum.

REDER, L. M. (1982). Plausibility judgments vs fact retrieval: Efficient strategies for question-answering. Psychological Review, 89, 250-280.

Smith, E. E., Adams, N., \& Schorr, D. (1978). Fact retrieval and the paradox of interference. Cognitive Psychology, 10, 438-464.

(Manuscript received for publication March 14, 1984.) 